\title{
Possibility Intuitionistic Fuzzy Soft Expert Set Theory and Its Application in Decision Making
}

\author{
Ganeshsree Selvachandran ${ }^{1}$ and Abdul Razak Salleh ${ }^{2}$ \\ ${ }^{1}$ Department of Actuarial Science and Applied Statistics, Faculty of Business \& Information Science, UCSI University, \\ Jalan Menara Gading, 56000 Cheras, Kuala Lumpur, Malaysia \\ ${ }^{2}$ School of Mathematical Sciences, Faculty of Science and Technology, Universiti Kebangsaan Malaysia, \\ 43600 Bangi, Selangor DE, Malaysia \\ Correspondence should be addressed to Ganeshsree Selvachandran; ganeshsree86@yahoo.com
}

Received 19 December 2014; Revised 5 March 2015; Accepted 12 March 2015

Academic Editor: Ram U. Verma

Copyright (c) 2015 G. Selvachandran and A. R. Salleh. This is an open access article distributed under the Creative Commons Attribution License, which permits unrestricted use, distribution, and reproduction in any medium, provided the original work is properly cited.

\begin{abstract}
We propose the theory of possibility intuitionistic fuzzy soft expert theory and define some related concepts pertaining to this notion as well as the basic operations on this concept, namely, the complement, union, intersection, AND, and OR. The basic properties and relevant laws pertaining to this concept such as De Morgan's laws are proved. Lastly, a generalized algorithm is introduced and applied to the concept of possibility intuitionistic fuzzy soft expert sets in hypothetical decision making problem.
\end{abstract}

\section{Introduction}

Most real-life problems involve data with a high level of uncertainty and imprecision. Traditionally, classical mathematical theories such as fuzzy mathematics, probability theory, and interval mathematics are used to deal with uncertainties and fuzziness. However, all these theories have their inherent difficulties and weaknesses as pointed out by Molodtsov (see [1]) which make it unsuitable to be used to deal with uncertainties and fuzziness. This led to the introduction of the theory of soft sets by Molodtsov in 1999 (see [1]). He also demonstrated several applications of this theory in solving many practical problems in economics, engineering, social sciences, and medical sciences, among others. Maji et al. (see [2]) furthered the study of soft sets by initiating the concept of fuzzy soft sets and proceeded to use these theories in solving various decision making problems. Presently, research on the theoretical and application aspects of soft sets and its various generalizations are progressing rapidly.

Among the significant milestones in the development of the theory of soft sets and its generalizations is the introduction of the possibility value which indicates the degree of possibility of belongingness of the elements in the universal set (in addition to the degree of membership (and nonmembership) of each element) as well as the element of expert sets which enables the users to know the opinion of all the experts in one model without the need for any operations. These new aspects have further improved the theory of soft sets and made it better suited to be used in solving decision making problems, especially when used with the more accurate generalizations of soft sets such as fuzzy soft sets and intuitionistic fuzzy soft sets. Both these aspects were established by Alkhazaleh and Salleh (see [3]) and Alkhazaleh et al. (see [4]) who introduced the concept of soft expert sets and possibility fuzzy soft sets, respectively. The duo of Alkhazaleh and Salleh then proceeded to introduce the notion of fuzzy soft expert sets and possibility intuitionistic fuzzy soft sets (see [5] and [6], resp.) and gave the application of these concepts in decision making and medical diagnosis problems. Hassan and Alhazaymeh introduced the theory of vague soft expert sets and possibility vague soft sets (see [7] and [8], resp.) and used it to solve decision making and medical diagnosis problems. Subsequently, the aspects of the degree of possibility and expert sets were attached to and/or simultaneously added to existing generalizations of soft sets such as the case in the establishment of possibility fuzzy soft expert 
sets by Bashir and Salleh (see [9]) and possibility vague soft expert set theory by Selvachandran (see [10]).

In [6], the authors introduced the concept of possibility intuitionistic fuzzy soft sets as a generalization and improvement of the concept of possibility fuzzy soft sets. In this paper, we establish the notion of possibility intuitionistic fuzzy soft expert sets as an improvement to $[6,10]$. In our study, a degree of possibility of each element in the universal set of elements is attached to the parameterization of intuitionistic fuzzy sets while defining an intuitionistic fuzzy soft set. Furthermore, the opinion of a set of experts is also given to enable the users to know the opinion of all the experts without the need for any operations. This results in a significantly better and improved generalization of intuitionistic fuzzy soft sets which would in turn produce more accurate results when applied to decision making problems.

The rest of this paper is organized as follows. First some relevant background information pertaining to soft sets, intuitionistic fuzzy sets, intuitionistic fuzzy soft sets, soft expert sets, and possibility intuitionistic fuzzy soft sets is presented in Section 2. In Section 3, we present a brief comparison between the concepts of intuitionistic fuzzy sets, intervalvalued fuzzy sets, and bipolar-valued fuzzy sets. In Section 4, the definition of possibility intuitionistic fuzzy soft expert sets (PIFSES) and its related concepts are proposed. In Section 5, some basic operations on PIFSES, namely, the complement, union, intersection, AND, and OR, are introduced and its properties are studied and proved. In Section 6, an explicit algorithm is introduced and is applied to the proposed PIFSES to solve a decision making problem. The conclusion of the study is stated in Section 7.

\section{Preliminaries}

In this section, we recall some definitions and properties pertaining to soft sets, intuitionistic fuzzy sets, intuitionistic fuzzy soft sets, soft expert sets, and possibility intuitionistic fuzzy soft sets.

Definition 1 (see [1]). A pair $(F, A)$ is called a soft set over $U$, where $F$ is a mapping given by $F: A \rightarrow P(U)$. In other words, a soft set over $U$ is a parameterized family of subsets of the universe $U$. For $\varepsilon \in A, F(\varepsilon)$ may be considered as the set of $\varepsilon$-elements of the soft set $(F, A)$ or as the $\varepsilon$-approximate elements of the soft set.

Definition 2 (see [11]). An intuitionistic fuzzy set $A$ defined over a universe of discourse $U$ is an object in the following form:

$$
A=\left\{\left\langle x, \mu_{A}(x), v_{A}(x)\right\rangle: x \in U\right\},
$$

where the functions $\mu_{A}: U \rightarrow[0,1]$ and $\nu_{A}: U \rightarrow[0,1]$ are the membership function and nonmembership function, respectively, of every element $x \in U$ to set $A$ and $0 \leq$ $\mu_{A}(x)+\nu_{A}(x) \leq 1$ for every $x \in U$. In the event that $0 \leq$ $\mu_{A}(x)+v_{A}(x)<1$, there is a degree of uncertainty that exists for element $x$ with respect to set $A$. This degree of uncertainty denoted as $\pi_{A}(x)$ is defined as $\pi_{A}(x)=1-\mu_{A}(x)-\nu_{A}(x)$. In general, a high degree of uncertainty implies that there are a lot things that are unknown about element $x$ with respect to set $A$.

From now on, let $A$ and $B$ be intuitionistic fuzzy sets defined over a universal set $U$ and are as defined below:

$$
\begin{aligned}
& A=\left\{\left\langle x, \mu_{A}(x), v_{A}(x)\right\rangle: x \in U\right\}, \\
& B=\left\{\left\langle x, \mu_{B}(x), v_{B}(x)\right\rangle: x \in U\right\} .
\end{aligned}
$$

Definition 3 (see [11]). The subset and equality of two intuitionistic fuzzy sets $A$ and $B$ are as defined below:

(a) $A \subset B \leftrightarrow \mu_{A}(x) \leq \mu_{B}(x)$ and $\nu_{A}(x) \geq \nu_{B}(x)$ for all $x \in U$

(b) $A=B \leftrightarrow A \subset B$ and $B \subset A$.

Definition 4 (see [11]). The complement, union, and intersection of two intuitionistic fuzzy sets $A$ and $B$ are as defined below:

(a) $\bar{A}=\left\{\left\langle x, v_{A}(x), \mu_{A}(x)\right\rangle: x \in U\right\}$

(b) $A \cup B=\left\{\left\langle x, \max \left(\mu_{A}(x), \mu_{B}(x)\right), \min \left(\nu_{A}(x), \nu_{B}(x)\right)\right\rangle\right.$ : $x \in U\}$

(c) $A \cap B=\left\{\left\langle x, \min \left(\mu_{A}(x), \mu_{B}(x)\right), \max \left(\nu_{A}(x), \nu_{B}(x)\right)\right\rangle\right.$ : $x \in U\}$.

Definition 5 (see [11]). The support of set $A$ is a classical set that consists of all elements of $U$ with nonzero membership values in $A$;

$$
\text { Supp } A=\left\{x \in U: \mu_{A}(x) \neq 0\right\} .
$$

Definition 6 (see [11]). An intuitionistic fuzzy set $A$ is said to be a null intuitionistic fuzzy set if $\operatorname{Supp} A=0$; that is, $\mu_{A}(x)=0$ for all $x \in U$ although $\nu_{A}(x) \neq 0$ for any $x \in U$.

Definition 7 (see [11]). An intuitionistic fuzzy set $A$ is said to be an absolute intuitionistic fuzzy set if $\operatorname{Supp} A \neq 0$; that is, $\mu_{A}(x) \neq 0$ for all $x \in U$ although $\nu_{A}(x)=0$ for any $x \in U$.

Definition 8 (see [12]). Consider $U$ and $E$ as a universe set and a set of parameters, respectively. Let $P(U)$ denote the set of all intuitionistic fuzzy sets of $U$. Let $A \subseteq E$. A pair $(F, E)$ is an intuitionistic fuzzy soft set over $U$, where $F$ is a mapping given by $F: A \rightarrow P(U)$.

Definition 9 (see $[13])$. Let $(F, A)$ and $(G, B)$ be two intuitionistic fuzzy soft sets on the same universal set $U$. Then the union operation of $(F, A)$ and $(G, B)$, denoted by $(F, A) \widetilde{\cup}(G$, $B)$, is an intuitionistic fuzzy soft set defined as $(F, A) \widetilde{\cup}(G, B)=$ $(H, C)$, where $C=A \cup B$ and $\forall \varepsilon \in C$,

$$
H(\alpha)= \begin{cases}F(\varepsilon) & \varepsilon \in A-B, \\ G(\varepsilon) & \varepsilon \in B-A, \\ F(\varepsilon) \cup G(\varepsilon) & \varepsilon \in A \cap B,\end{cases}
$$

where $U$ is the basic union. 
Definition 10 (see [13]). Let $(F, A)$ and $(G, B)$ be two intuitionistic fuzzy soft sets on the same universal set $U$. Then the intersection operation of $(F, A)$ and $(G, B)$, denoted by $(F, A) \widetilde{\cap}(G$, $B)$, is an intuitionistic fuzzy soft set defined as $(F, A) \widetilde{\cap}(G, B)=$ $(H, C)$, where $C=A \cup B$ and $\forall \varepsilon \in C$,

$$
H(\alpha)= \begin{cases}F(\varepsilon) & \varepsilon \in A-B, \\ G(\varepsilon) & \varepsilon \in B-A, \\ F(\varepsilon) \cap G(\varepsilon) & \varepsilon \in A \cap B,\end{cases}
$$

where $\cap$ is the basic intersection.

From now on, let $U$ be a universe, let $E$ be a set of parameters, let $X$ be a set of experts (agents), and let $O$ be a set of opinions, $Z=E \times X \times O$ and $A \subseteq Z$.

Definition 11 (see [3]). A pair $(F, A)$ is called a soft expert set over $U$, where $F$ is a mapping given by

$$
F: A \longrightarrow P(U),
$$

where $P(U)$ denotes the power set of $U$.

Definition 12 (see [3]). The complement of a soft expert set $(F, A)$ is denoted by $(F, A)^{c}$ and is defined by $(F, A)^{c}=\left(F^{c}, \sim\right.$ $A)$, where $F^{c}: \sim A \rightarrow P(U)$ is a mapping given by $F^{c}(a)=$ $U-F(\sim a)$, for all $a \in \sim A$.

Definition 13 (see [6]). Let $U=\left\{x_{1}, x_{2}, x_{3}, \ldots, x_{n}\right\}$ be a universe and let $E=\left\{e_{1}, e_{2}, e_{3}, \ldots, e_{m}\right\}$ be a set of parameters. The pair $(U, E)$ will be called a soft universe. Let $F: E \rightarrow$ $(I \times I)^{U} \times I^{U}$, where $(I \times I)^{U}$ is the collection of all intuitionistic fuzzy subsets of $U$ and $I^{U}$ is the collection of all fuzzy subsets of $U$. Let $p$ be a fuzzy subset of $E$, that is, $p: E \rightarrow I^{U}$, and let $F_{p}: E \rightarrow(I \times I)^{U} \times I^{U}$ be a function defined as follows:

$$
\begin{aligned}
& F_{p}(e)=(F(e)(x), p(e)(x)), \quad \text { where } \\
& F(e)(x)=(\mu(x), \nu(x)) \quad \forall x \in U .
\end{aligned}
$$

Then $F_{p}$ is called a possibility intuitionistic fuzzy soft set (PIFSS) over the soft universe $(U, E)$.

\section{Comparison of Intuitionistic Fuzzy Sets, Interval-Valued Fuzzy Sets, and Bipolar-Valued Fuzzy Sets}

In this paper, we establish the notion of possibility intuitionistic fuzzy soft expert sets (PIFSES) as a generalization and improvement to $[6,10]$. The concept of intuitionistic fuzzy sets (IFS) which are used to derive the concept of PIFSES is a generalization of fuzzy sets, albeit a more superior one. IFS are commonly thought to be similar to the notion of interval-valued fuzzy sets (IVFS) and bipolar-valued fuzzy sets (BVFS). However, in reality these three generalizations of fuzzy sets are significantly different from one another. The concepts of IFS, IVFS, and BVFS are all extensions of the fuzzy set theory. The definitions of IVFS and BVFS are as given below while the definition of IFS has been given in Definition 2.

Definition 14 (see [14]). An interval-valued fuzzy set A defined over a universe of discourse $U$ is an object in the following form:

$$
\begin{aligned}
& A=\left\{\left\langle x, \mu_{A}(x)\right\rangle: x \in U\right\} \\
& \mu_{A}(x): U \longrightarrow P([0,1]),
\end{aligned}
$$

where $\mu_{A}(x)$ is a closed interval in $[0,1]$ for each $x \in U$ and represents the degree of membership of the elements in set $A$ with respect to the criteria that is being studied.

Definition 15 (see [15]). A bipolar-valued fuzzy set A defined over a universe of discourse $U$ is an object in the following form:

$$
\begin{aligned}
A= & \left\{\left\langle x, \mu_{A}^{P}(x), \mu_{A}^{N}\right\rangle: x \in U\right\}, \\
& \mu_{A}^{P}(x): U \longrightarrow[0,1], \\
& \mu_{A}^{N}(x): U \longrightarrow[-1,0],
\end{aligned}
$$

where $\mu_{A}^{P}(x)$ is the positive membership degree and denotes the degree of satisfaction of an element $x$ to the property corresponding to set $A$ and $\mu_{A}^{N}(x)$ is the negative membership degree and denotes the degree of satisfaction of $x$ to some implicit counter-property corresponding to set $A$.

IFS are characterized by a membership function and a nonmembership function which describes the degree of belongingness and nonbelongingness of the elements in a set with respect to certain criteria, respectively. They are a representation to express the uncertainty in assigning membership degrees to elements. IVFS are characterized by membership degrees of elements which can be intervals of real numbers in $[0,1]$. This is done so as to better express some uncertainties in assigning membership degrees. The larger the interval, the higher the degree of uncertainty in assigning the membership degree for an element (see [14]). BVFS, on the other hand, are an extension of fuzzy sets whose membership degree range is enlarged from the interval $[0,1]$ to $[-1,1]$. In BVFS, a membership degree of 0 means that the elements are irrelevant to the corresponding property, the membership degrees on $(0,1]$ indicate that the elements somewhat satisfy the property and the membership degrees on $[-1,0)$ indicate that the elements somewhat satisfy the implicit counter-property (see [15]). The BVFS has major advantages over many of the other generalizations of fuzzy sets and has the ability to represent the double-sided nature of human perception and cognition. However, in most decision making problems, we are often more concerned with whether an element satisfies or does not satisfy particular criteria or property and, specifically, the extent to which the element satisfies or does not satisfy the corresponding criteria, instead of whether an element satisfies the corresponding property and counter-property of the criteria that is being studied. As such, the concepts of 
PIFSES which are derived using the concept of IFS are better suited and more relevant to use in decision making problems as opposed to BVFS.

\section{Possibility Intuitionistic Fuzzy Soft Expert Sets}

In this section, the notion of possibility intuitionistic fuzzy soft expert sets (PIFSES) is established and the properties of this concept are studied and discussed.

From now on, let $U$ be universal set of elements, let $E$ be a set of parameters, let $X$ be a set of experts (agents), and let $Q$ be a set of opinions, $Z=E \times X \times Q$ and $A \subseteq Z$.

Definition 16. Let $U=\left\{u_{1}, u_{2}, u_{3}, \ldots, u_{n}\right\}$ be a universal set of elements, let $E=\left\{e_{1}, e_{2}, e_{3}, \ldots, e_{m}\right\}$ be a universal set of parameters, let $X=\left\{x_{1}, x_{2}, x_{3}, \ldots, x_{i}\right\}$ be a set of experts (agents), and let $Q=\{1=$ agree, $0=$ disagree $\}$ be a set of opinions. Let $Z=\{E \times X \times Q\}$ and $A \subseteq Z$. Then the pair $(U, Z)$ is called a soft universe. Let $F: Z \rightarrow I^{U}$ and $p$ be an intuitionistic fuzzy subset of $Z$ defined as $p: Z \rightarrow I^{U}$, where $I^{U}$ denotes the collection of all intuitionistic fuzzy subsets of $U$. Suppose $F_{p}: Z \rightarrow I^{U} \times I^{U}$ to be a function defined as

$$
F_{p}(z)=\left(F(z)\left(u_{i}\right), p(z)\left(u_{i}\right)\right), \quad \forall u_{i} \in U
$$

Then $F_{p}$ is called a possibility intuitionistic fuzzy soft expert set (denoted as PIFSES for simplicity) over the soft universe $(U, Z)$.

For each $z_{i} \in Z, F_{p}\left(z_{i}\right)=\left(F\left(z_{i}\right)\left(u_{i}\right), p\left(z_{i}\right)\left(u_{i}\right)\right)$, where $F\left(z_{i}\right)$ represents the degree of belongingness and nonbelongingness of the elements of $U$ in $F\left(z_{i}\right)$ and $p\left(z_{i}\right)$ represents the degree of possibility of such belongingness. Hence $F_{p}\left(z_{i}\right)$ can be written as

$$
F_{p}\left(z_{i}\right)=\left\{\left(\frac{u_{i}}{F\left(z_{i}\right)\left(u_{i}\right)}\right), p\left(z_{i}\right)\left(u_{i}\right)\right\}, \quad \text { for } i=1,2,3, \ldots,
$$

where $F\left(z_{i}\right)\left(u_{i}\right)=\left\langle\mu_{F\left(z_{i}\right)}\left(u_{i}\right), \nu_{F\left(z_{i}\right)}\left(u_{i}\right)\right\rangle$, with $\mu_{F\left(z_{i}\right)}\left(u_{i}\right)$ and $v_{F\left(z_{i}\right)}\left(u_{i}\right)$ representing the membership function and nonmembership function of each of the elements $u_{i} \in U$, respectively.

Often the PIFSES $\left(F_{p}, Z\right)$ can be written simply as $F_{p}$. If $A \subseteq Z$, it is also possible to have a PIFSES $\left(F_{p}, A\right)$.

Note. For the sake of simplicity, in this paper, it is assumed that the set of opinions only consist of two values, namely, agree and disagree. However, it is possible to include other options for the set of opinions, including more specific opinions.

Example 17. Let $U=\left\{u_{1}, u_{2}, u_{3}\right\}$ be a set of elements, let $E=$ $\left\{e_{1}, e_{2}\right\}$ be a set of decision parameters, where $e_{i}(i=1,2,3)$ denotes the parameters $E=\left\{e_{1}=\right.$ beautiful, $e_{2}=$ cheap $\}$, and let $X=\left\{x_{1}, x_{2}\right\}$ be a set of experts. Suppose that $F_{p}: Z \rightarrow$ $I^{U} \times I^{U}$ is a function defined as follows:

$$
\begin{aligned}
& F_{p}\left(e_{1}, x_{1}, 1\right) \\
& =\left\{\left(\frac{u_{1}}{\langle 0.8,0.1\rangle}, 0.3\right),\left(\frac{u_{2}}{\langle 0.4,0.5\rangle}, 0.2\right),\left(\frac{u_{3}}{\langle 0.6,0.4\rangle}, 0.3\right)\right\}, \\
& F_{p}\left(e_{2}, x_{1}, 1\right) \\
& =\left\{\left(\frac{u_{1}}{\langle 0.6,0.3\rangle}, 0.6\right),\left(\frac{u_{2}}{\langle 0.1,0.9\rangle}, 0.8\right),\left(\frac{u_{3}}{\langle 0.9,0.05\rangle}, 0.5\right)\right\}, \\
& F_{p}\left(e_{1}, x_{2}, 1\right) \\
& =\left\{\left(\frac{u_{1}}{\langle 0.4,0.6\rangle}, 0.4\right),\left(\frac{u_{2}}{\langle 0.5,0.5\rangle}, 0.3\right),\left(\frac{u_{3}}{\langle 0.9,0.1\rangle}, 0.7\right)\right\}, \\
& F_{p}\left(e_{2}, x_{2}, 1\right) \\
& =\left\{\left(\frac{u_{1}}{\langle 0,1\rangle}, 0.9\right),\left(\frac{u_{2}}{\langle 0.3,0.4\rangle}, 0.8\right),\left(\frac{u_{3}}{\langle 0.2,0.75\rangle}, 0.15\right)\right\}, \\
& F_{p}\left(e_{1}, x_{1}, 0\right) \\
& =\left\{\left(\frac{u_{1}}{\langle 0.2,0.8\rangle}, 0.5\right),\left(\frac{u_{2}}{\langle 0.3,0.7\rangle}, 0\right),\left(\frac{u_{3}}{\langle 0.9,0\rangle}, 0.1\right)\right\}, \\
& F_{p}\left(e_{2}, x_{1}, 0\right) \\
& =\left\{\left(\frac{u_{1}}{\langle 0,0.82\rangle}, 0.75\right),\left(\frac{u_{2}}{\langle 0.3,0.7\rangle}, 0.2\right),\left(\frac{u_{3}}{\langle 0.8,0.2\rangle}, 0.6\right)\right\}, \\
& F_{p}\left(e_{1}, x_{2}, 0\right) \\
& =\left\{\left(\frac{u_{1}}{\langle 0.8,0.1\rangle}, 0.62\right),\left(\frac{u_{2}}{\langle 1,0\rangle}, 0.25\right),\left(\frac{u_{3}}{\langle 0.3,0.55\rangle}, 0.9\right)\right\}, \\
& F_{p}\left(e_{2}, x_{2}, 0\right) \\
& =\left\{\left(\frac{u_{1}}{\langle 0.3,0.5\rangle}, 0.55\right),\left(\frac{u_{2}}{\langle 0.7,0.3\rangle}, 1\right),\left(\frac{u_{3}}{\langle 1,0\rangle}, 0.99\right)\right\} .
\end{aligned}
$$

Then we can view the possibility intuitionistic fuzzy soft expert set $\left(F_{p}, Z\right)$ as consisting of the following collection of approximations:

$$
\begin{aligned}
& \left(F_{p}, Z\right) \\
& =\left\{\left(e_{1}, x_{1}, 1\right)=\left\{\left(\frac{u_{1}}{\langle 0.8,0.1\rangle}, 0.3\right),\right.\right. \\
& \left.\left.\quad\left(\frac{u_{2}}{\langle 0.4,0.5\rangle}, 0.2\right),\left(\frac{u_{3}}{\langle 0.6,0.4\rangle}, 0.3\right)\right\}\right\}, \\
& \left\{\left(e_{2}, x_{1}, 1\right)=\left\{\left(\frac{u_{1}}{\langle 0.6,0.3\rangle}, 0.6\right),\left(\frac{u_{2}}{\langle 0.1,0.9\rangle}, 0.8\right),\right.\right. \\
& \left.\left.\quad\left(\frac{u_{3}}{\langle 0.9,0.05\rangle}, 0.5\right)\right\}\right\},
\end{aligned}
$$




$$
\begin{aligned}
& \left\{\left(e_{1}, x_{2}, 1\right)=\left\{\left(\frac{u_{1}}{\langle 0.4,0.6\rangle}, 0.4\right),\left(\frac{u_{2}}{\langle 0.5,0.5\rangle}, 0.3\right)\right. \text {, }\right. \\
& \left.\left.\left(\frac{u_{3}}{\langle 0.9,0.1\rangle}, 0.7\right)\right\}\right\} \text {, } \\
& \left\{\left(e_{2}, x_{2}, 1\right)=\left\{\left(\frac{u_{1}}{\langle 0,1\rangle}, 0.9\right),\left(\frac{u_{2}}{\langle 0.3,0.4\rangle}, 0.8\right)\right. \text {, }\right. \\
& \left.\left.\left(\frac{u_{3}}{\langle 0.2,0.75\rangle}, 0.15\right)\right\}\right\} \text {, } \\
& \left\{\left(e_{1}, x_{1}, 0\right)=\left\{\left(\frac{u_{1}}{\langle 0.2,0.8\rangle}, 0.5\right),\left(\frac{u_{2}}{\langle 0.3,0.7\rangle}, 0\right)\right. \text {, }\right. \\
& \left.\left.\left(\frac{u_{3}}{\langle 0.9,0\rangle}, 0.1\right)\right\}\right\} \text {, } \\
& \left\{\left(e_{2}, x_{1}, 0\right)=\left\{\left(\frac{u_{1}}{\langle 0,0.82\rangle}, 0.75\right),\left(\frac{u_{2}}{\langle 0.3,0.7\rangle}, 0.2\right)\right. \text {, }\right. \\
& \left.\left.\left(\frac{u_{3}}{\langle 0.8,0.2\rangle}, 0.6\right)\right\}\right\} \text {, } \\
& \left\{\left(e_{1}, x_{2}, 0\right)=\left\{\left(\frac{u_{1}}{\langle 0.8,0.1\rangle}, 0.62\right),\left(\frac{u_{2}}{\langle 1,0\rangle}, 0.25\right)\right. \text {, }\right. \\
& \left.\left.\left(\frac{u_{3}}{\langle 0.3,0.55\rangle}, 0.9\right)\right\}\right\} \text {, } \\
& \left\{\left(e_{2}, x_{2}, 0\right)=\left\{\left(\frac{u_{1}}{\langle 0.3,0.5\rangle}, 0.55\right),\left(\frac{u_{2}}{\langle 0.7,0.3\rangle}, 1\right)\right. \text {, }\right. \\
& \left.\left.\left(\frac{u_{3}}{\langle 1,0\rangle}, 0.99\right)\right\}\right\} \text {. }
\end{aligned}
$$

Then $\left(F_{p}, Z\right)$ is a possibility intuitionistic fuzzy soft expert set over the soft universe $(U, Z)$.

Definition 18. Let $\left(F_{p}, A\right)$ and $\left(G_{q}, B\right)$ be PIFSES over a soft universe $(U, Z)$. Then $\left(F_{p}, A\right)$ is said to be a possibility intuitionistic fuzzy soft expert subset (PIFSE subset) of $\left(G_{q}, B\right)$ if $A \subseteq B$, and for all $\varepsilon \in A$, the following conditions are satisfied:

(i) $p(\varepsilon)$ is an intuitionistic fuzzy subset of $q(\varepsilon)$,

(ii) $F(\varepsilon)$ is an intuitionistic fuzzy subset of $G(\varepsilon)$.

This relationship is denoted as $\left(F_{p}, A\right) \subseteq\left(G_{q}, B\right)$. In this case, $\left(G_{q}, B\right)$ is called a possibility intuitionistic fuzzy soft expert superset (PIFSE superset) of $\left(F_{p}, A\right)$.

Definition 19. Let $\left(F_{p}, A\right)$ and $\left(G_{q}, B\right)$ be PIFSES over a soft universe $(U, Z)$. Then $\left(F_{p}, A\right)$ and $\left(G_{q}, B\right)$ are said to be equal if, for all $\varepsilon \in E$, the following conditions are satisfied:

(i) $p(\varepsilon)$ is equal to $q(\varepsilon)$,

(ii) $F(\varepsilon)$ is equal to $G(\varepsilon)$.

In other words, $\left(F_{p}, A\right)=\left(G_{q}, B\right)$ if $\left(F_{p}, A\right)$ is a PIFSE subset of $\left(G_{q}, B\right)$ and $\left(G_{q}, B\right)$ is a PIFSE subset of $\left(F_{p}, A\right)$.
Definition 20. A PIFSES $\left(F_{p}, A\right)$ is said to be a null possibility intuitionistic fuzzy soft expert set, denoted by $\left(\widetilde{\phi}_{p}, A\right)$ and defined as

$$
\left(\tilde{\phi}_{p}, A\right)=(F(\alpha), p(\alpha)), \quad \forall \alpha \in Z,
$$

where $F(\alpha)=\langle 0,1\rangle$, that is, $\mu_{F(\alpha)}=0$ and $\nu_{F(\alpha)}=1$, and $p(\alpha)=0$ for all $\alpha \in Z$.

Definition 21. A PIFSES $\left(F_{p}, A\right)$ is said to be an absolute possibility intuitionistic fuzzy soft expert set, denoted by $\left(F_{p}, A\right)_{\mathrm{Abs}}$ and defined as

$$
\left(F_{p}, A\right)_{\mathrm{Abs}}=(F(\alpha), p(\alpha)), \quad \forall \alpha \in Z,
$$

where $F(\alpha)=\langle 1,0\rangle$, that is, $\mu_{F(\alpha)}=1$ and $\nu_{F(\alpha)}=0$, and $p(\alpha)=1$ for all $\alpha \in Z$.

Definition 22. Let $\left(F_{p}, A\right)$ be a PIFSES over a soft universe $(U, Z)$. An agree-possibility intuitionistic fuzzy soft expert set (agree-PIFSES) over $U$, denoted as $\left(F_{p}, A\right)_{1}$, is a possibility intuitionistic fuzzy soft expert subset of $\left(F_{p}, A\right)$ which is defined as

$$
\left(F_{p}, A\right)_{1}=(F(\alpha), p(\alpha)), \quad \text { where } \alpha \in E \times X \times\{1\} .
$$

Definition 23. Let $\left(F_{p}, A\right)$ be a PIFSES over a soft universe $(U, Z)$. A disagree-possibility intuitionistic fuzzy soft expert set (disagree-PIFSES) over $U$, denoted as $\left(F_{p}, A\right)_{0}$, is a possibility intuitionistic fuzzy soft expert subset of $\left(F_{p}, A\right)$ which is defined as

$$
\left(F_{p}, A\right)_{0}=(F(\alpha), p(\alpha)), \quad \text { where } \alpha \in E \times X \times\{0\} .
$$

\section{Basic Operations on Possibility Intuitionistic Fuzzy Soft Expert Sets}

In this section, we introduce some basic operations on PIFSES, namely, the complement, AND, OR, union, and intersection of PIFSES, and proceed to study some of the properties related to these operations.

Definition 24. Let $\left(F_{p}, A\right)$ be a PIFSES over a soft universe $(U, Z)$. Then the complement of $\left(F_{p}, A\right)$, denoted by $\left(F_{p}, A\right)^{c}$, is defined as

$$
\left(F_{p}, A\right)^{c}=(\widetilde{c}(F(\alpha)), c(p(\alpha))), \quad \forall a \in A,
$$

where $\widetilde{c}$ is an intuitionistic fuzzy complement and $c$ is a fuzzy complement.

Example 25. Consider the PIFSES $\left(F_{p}, Z\right)$ over a soft universe $(U, Z)$ as given in Example 17. By using the basic fuzzy 
complement for $p(\alpha)$ and the intuitionistic fuzzy complement for $F(\alpha)$, we obtain $\left(F_{p}, Z\right)^{c}$ which is defined as

$$
\begin{aligned}
& \left(F_{p}, Z\right)^{c} \\
& =\left\{\left(e_{1}, x_{1}, 1\right)=\left\{\left(\frac{u_{1}}{\langle 0.1,0.8\rangle}, 0.3\right)\right. \text {, }\right. \\
& \left.\left.\left(\frac{u_{2}}{\langle 0.5,0.4\rangle}, 0.2\right),\left(\frac{u_{3}}{\langle 0.4,0.6\rangle}, 0.3\right)\right\}\right\}, \\
& \left\{\left(e_{2}, x_{1}, 1\right)=\left\{\left(\frac{u_{1}}{\langle 0.3,0.6\rangle}, 0.6\right),\left(\frac{u_{2}}{\langle 0.9,0.1\rangle}, 0.8\right)\right. \text {, }\right. \\
& \left.\left.\left(\frac{u_{3}}{\langle 0.05,0.9\rangle}, 0.5\right)\right\}\right\} \text {, } \\
& \left\{\left(e_{1}, x_{2}, 1\right)=\left\{\left(\frac{u_{1}}{\langle 0.6,0.4\rangle}, 0.4\right),\left(\frac{u_{2}}{\langle 0.5,0.5\rangle}, 0.3\right)\right. \text {, }\right. \\
& \left.\left.\left(\frac{u_{3}}{\langle 0.1,0.9\rangle}, 0.7\right)\right\}\right\} \text {, } \\
& \left\{\left(e_{2}, x_{2}, 1\right)=\left\{\left(\frac{u_{1}}{\langle 1,0\rangle}, 0.9\right),\left(\frac{u_{2}}{\langle 0.4,0.3\rangle}, 0.8\right),\right.\right. \\
& \left.\left.\left(\frac{u_{3}}{\langle 0.75,0.2\rangle}, 0.15\right)\right\}\right\} \\
& \left\{\left(e_{1}, x_{1}, 0\right)=\left\{\left(\frac{u_{1}}{\langle 0.8,0.2\rangle}, 0.5\right),\left(\frac{u_{2}}{\langle 0.7,0.3\rangle}, 0\right),\right.\right. \\
& \left.\left.\left(\frac{u_{3}}{\langle 0,0.9\rangle}, 0.1\right)\right\}\right\} \text {, } \\
& \left\{\left(e_{2}, x_{1}, 0\right)=\left\{\left(\frac{u_{1}}{\langle 0.82,0\rangle}, 0.75\right),\left(\frac{u_{2}}{\langle 0.7,0.3\rangle}, 0.2\right)\right. \text {, }\right. \\
& \left.\left.\left(\frac{u_{3}}{\langle 0.2,0.8\rangle}, 0.6\right)\right\}\right\} \\
& \left\{\left(e_{1}, x_{2}, 0\right)=\left\{\left(\frac{u_{1}}{\langle 0.1,0.8\rangle}, 0.62\right),\left(\frac{u_{2}}{\langle 0,1\rangle}, 0.25\right),\right.\right. \\
& \left.\left.\left(\frac{u_{3}}{\langle 0.55,0.3\rangle}, 0.9\right)\right\}\right\} \text {, } \\
& \left\{\left(e_{2}, x_{2}, 0\right)=\left\{\left(\frac{u_{1}}{\langle 0.5,0.3\rangle}, 0.55\right),\left(\frac{u_{2}}{\langle 0.3,0.7\rangle}, 1\right)\right. \text {, }\right. \\
& \left.\left.\left(\frac{u_{3}}{\langle 0,1\rangle}, 0.99\right)\right\}\right\} \text {. }
\end{aligned}
$$

Proposition 26. Let $\left(F_{p}, A\right)$ be a PIFSES over a soft universe $(U, Z)$. Then the following property holds true:

$$
\left(\left(F_{p}, A\right)^{c}\right)^{c}=\left(F_{p}, A\right)
$$

Proof. Suppose that $\left(F_{p}, A\right)$ is a PIFSES over a soft universe $(U, Z)$ defined as $\left(F_{p}, A\right)=(F(e), p(e))$. Now let $\left(F_{p}, A\right)^{c}=$
$\left(G_{q}, B\right)$. Then by Definition $24,\left(G_{q}, B\right)=(G(e), q(e))$ such that $G(e)=\widetilde{c}(F(e))$ and $q(e)=c(p(e))$. Thus it follows that

$$
\begin{aligned}
\left(G_{q}, B\right)^{c} & =(\widetilde{c}(G(e)), c(q(e)))=(\widetilde{c}(\widetilde{c}(F(e))), c(c(p(e)))) \\
& =(F(e), p(e))=\left(F_{p}, A\right) .
\end{aligned}
$$

Therefore $\left(\left(F_{p}, A\right)^{c}\right)^{c}=\left(G_{q}, B\right)^{c}=\left(F_{p}, A\right)$. Hence it is proven that $\left(\left(F_{p}, A\right)^{c}\right)^{c}=\left(F_{p}, A\right)$.

Definition 27. Let $\left(F_{p}, A\right)$ and $\left(G_{q}, B\right)$ be PIFSES over a soft universe $(U, Z)$. Then the union of $\left(F_{p}, A\right)$ and $\left(G_{q}, B\right)$, denoted by $\left(F_{p}, A\right) \widetilde{U}\left(G_{q}, B\right)$, is a PIFSES defined as $\left(F_{p}, A\right) \widetilde{U}$ $\left(G_{q}, B\right)=\left(H_{r}, C\right)$, where $C=A \cup B$ and

$$
\begin{aligned}
r(\alpha) & =\max (p(\alpha), q(\alpha)), \quad \forall \alpha \in C, \\
H(\alpha) & =F(\alpha) \widetilde{\cup} G(\alpha), \quad \forall \alpha \in C,
\end{aligned}
$$

where

$$
H(\alpha)= \begin{cases}F(\alpha) & \alpha \in A-B, \\ G(\alpha) & \alpha \in B-A, \\ \max (F(\alpha), G(\alpha)) & \alpha \in A \cap B .\end{cases}
$$

Proposition 28. Let $\left(F_{p}, A\right),\left(G_{q}, B\right)$, and $\left(H_{r}, C\right)$ be any three PVSESs over a soft universe $(U, Z)$. Then the following results hold true:

(i) $\left(F_{p}, A\right) \widetilde{U}\left(G_{q}, B\right)=\left(G_{q}, B\right) \widetilde{U}\left(F_{p}, A\right)$ (commutative law);

(ii) $\left(F_{p}, A\right) \widetilde{U}\left(\left(G_{q}, B\right) \widetilde{U}\left(H_{r}, A\right)\right)=\left(\left(F_{p}, A\right) \widetilde{U}\left(G_{q}, B\right)\right) \widetilde{U}\left(H_{r}\right.$, C) (associative law);

(iii) $\left(F_{p}, A\right) \widetilde{\cup}\left(F_{p}, A\right) \subseteq\left(F_{p}, A\right)$;

(iv) $\left(F_{p}, A\right) \widetilde{\cup}\left(\emptyset_{p}, A\right)=\left(F_{p}, A\right)$.

Proof. (i) Let $\left(F_{p}, A\right) \widetilde{\cup}\left(G_{q}, B\right)=\left(H_{r}, C\right)$. Then by Definition 27, for all $\alpha \in C$, we have

$$
\left(H_{r}, C\right)=(H(\alpha), r(\alpha))
$$

where $H(\alpha)=F(\alpha) \tilde{\cup} G(\alpha)$ and $r(\alpha)=\max (p(\alpha), q(\alpha))$. However, $H(\alpha)=F(\alpha) \widetilde{\cup} G(\alpha)=G(\alpha) \widetilde{\cup} F(\alpha)$ since the union of these sets is commutative by Definition 27. Also, $r(\alpha)=$ $\max (p(\alpha), q(\alpha))=\max (q(\alpha), p(\alpha))$. Therefore, $\left(H_{r}, C\right)=$ $\left(G_{q}, B\right) \widetilde{\cup}\left(F_{p}, A\right)$. Thus the union of two PIFSES is commutative; that is, $\left(F_{p}, A\right) \widetilde{\cup}\left(G_{q}, B\right)=\left(G_{q}, B\right) \widetilde{\cup}\left(F_{p}, A\right)$.

(ii) The proof is similar to the proof of part (i) and is therefore omitted.

(iii) The proof is straightforward and is therefore omitted.

(iv) The proof is straightforward and is therefore omitted.

Definition 29. Let $\left(F_{p}, A\right)$ and $\left(G_{q}, B\right)$ be PIFSES over a soft universe $(U, Z)$. Then the intersection of $\left(F_{p}, A\right)$ and $\left(G_{q}, B\right)$, 
denoted by $\left(F_{p}, A\right) \widetilde{\cap}\left(G_{q}, B\right)$, is PIFSES defined as $\left(F_{p}, A\right) \widetilde{\cap}\left(G_{q}\right.$, $B)=\left(H_{r}, C\right)$, where $C=A \cup B$ and

$$
\begin{aligned}
r(\alpha) & =\min (p(\alpha), q(\alpha)), \quad \forall \alpha \in C, \\
H(\alpha) & =F(\alpha) \widetilde{\cap} G(\alpha), \quad \forall \alpha \in C,
\end{aligned}
$$

where

$$
H(\alpha)= \begin{cases}F(\alpha) & \alpha \in A-B, \\ G(\alpha) & \alpha \in B-A \\ \min (F(\alpha), G(\alpha)) & \alpha \in A \cap B\end{cases}
$$

Proposition 30. Let $\left(F_{p}, A\right),\left(G_{q}, B\right)$, and $\left(H_{r}, C\right)$ be any three PIFSES over a soft universe $(U, Z)$. Then the following results hold true:

(i) $\left(F_{p}, A\right) \widetilde{\cap}\left(G_{q}, B\right)=\left(G_{q}, B\right) \widetilde{\cap}\left(F_{p}, A\right)$ (commutative law);

(ii) $\left(F_{p}, A\right) \widetilde{\cap}\left(\left(G_{q}, B\right) \widetilde{\cap}\left(H_{r}, C\right)\right)=\left(\left(F_{p}, A\right) \widetilde{\cap}\left(G_{q}, B\right)\right) \widetilde{\cap}\left(H_{r}\right.$, C) (associative law);

(iii) $\left(F_{p}, A\right) \widetilde{\cap}\left(F_{p}, A\right) \subseteq\left(F_{p}, A\right)$;

(iv) $\left(F_{p}, A\right) \widetilde{\cap}\left(\emptyset_{p}, A\right)=\left(\emptyset_{p}, A\right)$.

Proof. (i) The proof is similar to that of Proposition 28 (i) and is therefore omitted.

(ii) The proof is similar to the proof of part (i) and is therefore omitted.

(iii) The proof is straightforward and is therefore omitted.

(iv) The proof is straightforward and is therefore omitted.

Proposition 31. Let $\left(F_{p}, A\right),\left(G_{q}, B\right)$, and $\left(H_{r}, C\right)$ be any three PIFSES over a soft universe $(U, Z)$. Then the following results hold true:

(i) $\left(F_{p}, A\right) \widetilde{\cup}\left(\left(G_{q}, B\right) \widetilde{\cap}\left(H_{r}, C\right)\right)=\left(\left(F_{p}, A\right) \widetilde{\cup}\left(G_{q}, B\right)\right) \widetilde{\cap}\left(\left(F_{p}\right.\right.$, A) $\left.\widetilde{\cup}\left(H_{r}, C\right)\right)$;

(ii) $\left(F_{p}, A\right) \widetilde{\cap}\left(\left(G_{q}, B\right) \widetilde{\cup}\left(H_{r}, C\right)\right)=\left(\left(F_{p}, A\right) \widetilde{\cap}\left(G_{q}, B\right)\right) \widetilde{\cup}\left(\left(F_{p}\right.\right.$, A) $\left.\widetilde{\cap}\left(H_{r}, C\right)\right)$.

Proof. The proof is straightforward by Definitions 27 and 29 and is therefore omitted.

Proposition 32. Let $\left(F_{p}, A\right)$ and $\left(G_{q}, B\right)$ be any two PIFSES over a soft universe $(U, Z)$. Then De Morgan's laws hold true:

(i) $\left(\left(F_{p}, A\right) \widetilde{\cup}\left(G_{q}, B\right)\right)^{c}=\left(F_{p}, A\right)^{c} \widetilde{\cap}\left(G_{q}, B\right)^{c}$;

(ii) $\left(\left(F_{p}, A\right) \widetilde{\cap}\left(G_{q}, B\right)\right)^{c}=\left(F_{p}, A\right)^{c} \widetilde{\cup}\left(G_{q}, B\right)^{c}$.

Proof. (i) Let $\left(F_{p}, A\right)$ and $\left(G_{q}, B\right)$ be PIFSES over a soft universe $(U, Z)$ defined as $\left(F_{p}, A\right)=(F(\alpha), p(\alpha))$ for all $\alpha \in$ $A \subseteq Z$ and $\left(G_{q}, B\right)=(G(\alpha), q(\alpha))$ for all $\alpha \in B \subseteq Z$. Now, due to the commutative and associative properties of PIFSES, it follows that

$$
\begin{aligned}
\left(F_{p}, A\right)^{c} & \tilde{\cap}\left(G_{q}, B\right)^{c} \\
& =(F(\alpha), p(\alpha))^{c} \widetilde{\cap}(G(\alpha), q(\alpha))^{c} \\
& =(\widetilde{c}(F(\alpha)), c(p(\alpha))) \widetilde{\cap}(\widetilde{c}(G(\alpha)), c(q(\alpha))) \\
& =((\widetilde{c}(F(\alpha)) \widetilde{\cap} \widetilde{c}(G(\alpha))), \min (c(p(\alpha)), c(q(\alpha)))) \\
& =(\widetilde{c}(F(\alpha) \widetilde{\cup} G(\alpha)), c(\max (p(\alpha), q(\alpha)))) \\
& =\left(\left(F_{p}, A\right) \widetilde{\cup}\left(G_{q}, B\right)\right)^{c} .
\end{aligned}
$$

(ii) The proof is similar to the proof of part (i) and is therefore omitted.

Definition 33. Let $\left(F_{p}, A\right)$ and $\left(G_{q}, B\right)$ be PIFSES over a soft universe $(U, Z)$. Then " $\left(F_{p}, A\right)$ AND $\left(G_{q}, B\right)$," denoted by $\left(F_{p}, A\right) \widetilde{\wedge}\left(G_{q}, B\right)$, is a PIFSES defined by

$$
\left(F_{p}, A\right) \tilde{\wedge}\left(G_{q}, B\right)=\left(H_{r}, A \times B\right),
$$

where $\left(H_{r}, A \times B\right)=(H(\alpha, \beta), r(\alpha, \beta))$, such that $H(\alpha, \beta)=$ $F(\alpha) \cap G(\beta)$ and $r(\alpha, \beta)=\min (p(\alpha), q(\beta))$, for all $(\alpha, \beta) \epsilon$ $A \times B$ and $\cap$ represents the basic intersection.

Definition 34. Let $\left(F_{p}, A\right)$ and $\left(G_{q}, B\right)$ be PIFSES over a soft universe $(U, Z)$. Then " $\left(F_{p}, A\right)$ OR $\left(G_{q}, B\right)$," denoted by $\left(F_{p}, A\right) \widetilde{V}\left(G_{q}, B\right)$, is a PIFSES defined by

$$
\left(F_{p}, A\right) \widetilde{V}\left(G_{q}, B\right)=\left(H_{r}, A \times B\right),
$$

where $\left(H_{r}, A \times B\right)=(H(\alpha, \beta), r(\alpha, \beta))$, such that $H(\alpha, \beta)=$ $F(\alpha) \cup G(\beta)$ and $r(\alpha, \beta)=\max (p(\alpha), q(\beta))$, for all $(\alpha, \beta) \epsilon$ $A \times B$ and $\cup$ represents the basic union.

Proposition 35. Let $\left(\widetilde{F}_{\mu}, A\right),\left(\widetilde{G}_{\delta}, B\right)$, and $\left(\widetilde{H}_{\lambda}, C\right)$ be any three $P V S E S$ over a soft universe $(U, Z)$. Then the following properties hold true:

(i) $\left(F_{p}, A\right) \tilde{\wedge}\left(\left(G_{q}, B\right) \tilde{\wedge}\left(H_{r}, C\right)\right)=\left(\left(F_{p}, A\right) \tilde{\wedge}\left(G_{q}, B\right)\right) \tilde{\wedge}\left(H_{r}\right.$, $C)$;

(ii) $\left(F_{p}, A\right) \widetilde{V}\left(\left(G_{q}, B\right) \widetilde{V}\left(H_{r}, C\right)\right)=\left(\left(F_{p}, A\right) \widetilde{V}\left(G_{q}, B\right)\right) \widetilde{V}\left(H_{r}\right.$, $C)$;

(iii) $\left(F_{p}, A\right) \widetilde{\vee}\left(\left(G_{q}, B\right) \widetilde{\wedge}\left(H_{r}, C\right)\right)=\left(\left(F_{p}, A\right) \widetilde{V}\left(G_{q}, B\right)\right) \widetilde{\wedge}\left(\left(F_{p}\right.\right.$, A) $\left.\widetilde{V}\left(H_{r}, C\right)\right)$

(iv) $\left(F_{p}, A\right) \widetilde{\wedge}\left(\left(G_{q}, B\right) \widetilde{V}\left(H_{r}, C\right)\right)=\left(\left(F_{p}, A\right) \widetilde{\wedge}\left(G_{q}, B\right)\right) \widetilde{V}\left(\left(F_{p}\right.\right.$, A) $\left.\widetilde{\wedge}\left(H_{r}, C\right)\right)$.

Proof. The proofs are straightforward by Definitions 33 and 34 and are therefore omitted.

Note. The "AND" and "OR" operations are not commutative since generally $A \times B \neq B \times A$. 
Proposition 36. Let $\left(F_{p}, A\right)$ and $\left(G_{q}, B\right)$ be any two PIFSES over a soft universe $(U, Z)$. Then the following De Morgan's laws hold true:

(i) $\left(\left(F_{p}, A\right) \widetilde{\wedge}\left(G_{q}, B\right)\right)^{c}=\left(F_{p}, A\right)^{c} \widetilde{\vee}\left(G_{q}, B\right)^{c}$;

(ii) $\left(\left(F_{p}, A\right) \widetilde{V}\left(G_{q}, B\right)\right)^{c}=\left(F_{p}, A\right)^{c} \widetilde{\wedge}\left(G_{q}, B\right)^{c}$.

Proof. (i) Suppose that $\left(F_{p}, A\right)$ and $\left(G_{q}, B\right)$ are PIFSES over a soft universe $(U, Z)$ defined as

$$
\begin{array}{ll}
\left(F_{p}, A\right)=(F(\alpha), p(\alpha)) & \forall \alpha \in A \subseteq Z, \\
\left(G_{q}, B\right)=(G(\beta), q(\beta)) & \forall \beta \in B \subseteq Z .
\end{array}
$$

Then by Definitions 33 and 34, it follows that

$$
\begin{aligned}
\left(\left(F_{p}, A\right) \tilde{\wedge}\left(G_{q}, B\right)\right)^{c} \\
=((F(\alpha), p(\alpha)) \tilde{\wedge}(G(\beta), q(\beta)))^{c} \\
=(F(\alpha) \cap G(\beta), \min (p(\alpha), q(\beta)))^{c} \\
=(\widetilde{c}(F(\alpha) \cap G(\beta)), c(\min (p(\alpha), q(\beta)))) \\
=(\widetilde{c}(F(\alpha)) \cup \widetilde{c}(G(\beta)), \max (c(p(\alpha)), c(q(\beta)))) \\
=(F(\alpha), p(\alpha))^{c} \widetilde{\nabla}(G(\beta), q(\beta))^{c} \\
=\left(F_{p}, A\right)^{c} \widetilde{\nabla}\left(G_{q}, B\right)^{c} .
\end{aligned}
$$

(ii) The proof is similar to that of part (i) and is therefore omitted.

\section{Application of Possibility \\ Intuitionistic Fuzzy Soft Expert Sets in a Decision Making Problem}

In this section, we introduce a generalized algorithm which will be applied to the PIFSES model introduced in Section 4 and used to solve a hypothetical decision making problem.

Suppose that company $Y$ is looking to hire a person to fill in the vacancy for a position in their company. Out of all the people who applied for the position, three candidates were shortlisted and these three candidates form the universe of elements, $U=\left\{u_{1}, u_{2}, u_{3}\right\}$. The hiring committee consists of the hiring manager, head of department, and the HR director of the company and this committee is represented by the set $X=\{p, q, r\}$ (a set of experts) while the set $Q=\{1=$ agree, $0=$ disagree $\}$ represents the set of opinions of the hiring committee members. The hiring committee considers a set of parameters, $E=\left\{e_{1}, e_{2}, e_{3}, e_{4}\right\}$, where the parameters $e_{i}(i=1,2,3,4)$ represent the characteristics or qualities that the candidates are assessed on, namely, "relevant job experience," "excellent academic qualifications in the relevant field," "attitude and level of professionalism," and "technical knowledge," respectively. After interviewing all the three candidates and going through their certificates and other supporting documents, the hiring committee constructs the following PIFSES:

$$
\begin{aligned}
& \left(F_{p}, Z\right) \\
& =\left\{\left(e_{1}, p, 1\right)=\left\{\left(\frac{u_{1}}{\langle 0.4,0.6\rangle}, 0.3\right),\right.\right. \\
& \left.\left.\qquad\left(\frac{u_{2}}{\langle 0.5,0.5\rangle}, 0.2\right),\left(\frac{u_{3}}{\langle 0.9,0.1\rangle}, 0.3\right)\right\}\right\}, \\
& \left\{\left(e_{2}, p, 1\right)=\left\{\left(\frac{u_{1}}{\langle 0,0.95\rangle}, 0.9\right),\left(\frac{u_{2}}{\langle 0.3,0.65\rangle}, 0.8\right),\right.\right. \\
& \left.\left.\left(\frac{u_{3}}{\langle 0.2,0.7\rangle}, 0.15\right)\right\}\right\},
\end{aligned}
$$

$$
\begin{aligned}
& \left\{\left(e_{3}, p, 1\right)=\left\{\left(\frac{u_{1}}{\langle 0.4,0.6\rangle}, 0.1\right),\left(\frac{u_{2}}{\langle 0.5,0.3\rangle}, 0.8\right),\right.\right. \\
& \left.\left.\left(\frac{u_{3}}{\langle 0,0.4\rangle}, 0.3\right)\right\}\right\}, \\
& \left\{\left(e_{4}, p, 1\right)=\left\{\left(\frac{u_{1}}{\langle 0.15,0.85\rangle}, 0.9\right),\left(\frac{u_{2}}{\langle 1,0\rangle}, 0.5\right),\right.\right. \\
& \left.\left\{\left(\frac{u_{3}}{\langle 0,1\rangle}, 1\right)\right\}\right\}, \\
& \left\{\left(e_{1}, q, 1\right)=\left\{\left(\frac{u_{1}}{\langle 0.8,0.2\rangle}, 0.3\right),\left(\frac{u_{2}}{\langle 0.4,0.5\rangle}, 0.2\right),\right.\right. \\
& \left.\left.\left(\frac{u_{3}}{\langle 0.6,0.3\rangle}, 0.3\right)\right\}\right\},
\end{aligned}
$$

$$
\begin{aligned}
& \left\{\left(e_{2}, q, 1\right)=\left\{\left(\frac{u_{1}}{\langle 0.6,0.4\rangle}, 0.6\right),\left(\frac{u_{2}}{\langle 1,0\rangle}, 0.8\right),\right.\right. \\
& \left.\left.\left(\frac{u_{3}}{\langle 0.9,0\rangle}, 0.5\right)\right\}\right\} \text {, } \\
& \left\{\left(e_{3}, q, 1\right)=\left\{\left(\frac{u_{1}}{\langle 0.9,0.1\rangle}, 0.9\right),\left(\frac{u_{2}}{\langle 0.5,0.2\rangle}, 0.4\right)\right. \text {, }\right. \\
& \left.\left.\left(\frac{u_{3}}{\langle 0,0.9\rangle}, 0.5\right)\right\}\right\} \text {, } \\
& \left\{\left(e_{4}, q, 1\right)=\left\{\left(\frac{u_{1}}{\langle 0,0.8\rangle}, 0.7\right),\left(\frac{u_{2}}{\langle 0.2,0.65\rangle}, 0.25\right)\right. \text {, }\right. \\
& \left.\left.\left(\frac{u_{3}}{\langle 1,0\rangle}, 0.45\right)\right\}\right\} \text {, } \\
& \left\{\left(e_{1}, r, 1\right)=\left\{\left(\frac{u_{1}}{\langle 0.3,0.55\rangle}, 0.7\right),\left(\frac{u_{2}}{\langle 0.1,0.7\rangle}, 0.25\right)\right. \text {, }\right. \\
& \left.\left.\left(\frac{u_{3}}{\langle 0,1\rangle}, 0\right)\right\}\right\}, \\
& \left\{\left(e_{2}, r, 1\right)=\left\{\left(\frac{u_{1}}{\langle 0.25,0.45\rangle}, 0.6\right),\left(\frac{u_{2}}{\langle 0.6,0.25\rangle}, 0.95\right)\right. \text {, }\right. \\
& \left.\left.\left(\frac{u_{3}}{\langle 0.4,0.6\rangle}, 0.1\right)\right\}\right\},
\end{aligned}
$$




$$
\begin{aligned}
& \left\{\left(e_{3}, r, 1\right)=\left\{\left(\frac{u_{1}}{\langle 0.8,0.05\rangle}, 0.99\right),\left(\frac{u_{2}}{\langle 0.2,0.75\rangle}, 0.8\right),\right.\right. \\
& \left.\left.\left(\frac{u_{3}}{\langle 0.05,0.9\rangle}, 0.4\right)\right\}\right\} \text {, } \\
& \left\{\left(e_{1}, p, 0\right)=\left\{\left(\frac{u_{1}}{\langle 0.2,0.6\rangle}, 0.5\right),\left(\frac{u_{2}}{\langle 0.3,0.4\rangle}, 0\right),\right.\right. \\
& \left.\left.\left(\frac{u_{3}}{\langle 0.9,0\rangle}, 0.1\right)\right\}\right\} \text {, } \\
& \left\{\left(e_{3}, p, 0\right)=\left\{\left(\frac{u_{1}}{\langle 0,0.92\rangle}, 0.75\right),\left(\frac{u_{2}}{\langle 0.3,0.7\rangle}, 0.2\right),\right.\right. \\
& \left.\left.\left(\frac{u_{3}}{\langle 0.8,0.15\rangle}, 0.6\right)\right\}\right\} \text {, } \\
& \left\{\left(e_{4}, p, 0\right)=\left\{\left(\frac{u_{1}}{\langle 0.8,0\rangle}, 0.62\right),\left(\frac{u_{2}}{\langle 1,0\rangle}, 0.05\right),\right.\right. \\
& \left.\left.\left(\frac{u_{3}}{\langle 0.35,0.55\rangle}, 0.9\right)\right\}\right\} \text {, } \\
& \left\{\left(e_{1}, q, 0\right)=\left\{\left(\frac{u_{1}}{\langle 0.3,0.5\rangle}, 0.55\right),\left(\frac{u_{2}}{\langle 0.7,0.3\rangle}, 1\right),\right.\right. \\
& \left.\left.\left(\frac{u_{3}}{\langle 1,0\rangle}, 0.99\right)\right\}\right\} \text {, } \\
& \left\{\left(e_{2}, q, 0\right)=\left\{\left(\frac{u_{1}}{\langle 0.2,0.7\rangle}, 0.2\right),\left(\frac{u_{2}}{\langle 0.1,0.75\rangle}, 0.3\right),\right.\right. \\
& \left.\left.\left(\frac{u_{3}}{\langle 0.5,0.4\rangle}, 0.1\right)\right\}\right\} \text {, } \\
& \left\{\left(e_{3}, q, 0\right)=\left\{\left(\frac{u_{1}}{\langle 0,1\rangle}, 0.6\right),\left(\frac{u_{2}}{\langle 0.3,0.65\rangle}, 0.8\right)\right. \text {, }\right. \\
& \left.\left.\left(\frac{u_{3}}{\langle 0,0.1\rangle}, 1\right)\right\}\right\} \text {, } \\
& \left\{\left(e_{4}, q, 0\right)=\left\{\left(\frac{u_{1}}{\langle 1,0\rangle}, 1\right),\left(\frac{u_{2}}{\langle 0.4,0.5\rangle}, 0.4\right),\right.\right. \\
& \left.\left.\left(\frac{u_{3}}{\langle 0,0.5\rangle}, 0.9\right)\right\}\right\} \text {, } \\
& \left\{\left(e_{1}, r, 0\right)=\left\{\left(\frac{u_{1}}{\langle 0.3,0.5\rangle}, 0.4\right),\left(\frac{u_{2}}{\langle 0.55,0.3\rangle}, 0.7\right)\right. \text {, }\right. \\
& \left.\left.\left(\frac{u_{3}}{\langle 0.4,0.6\rangle}, 0.3\right)\right\}\right\} \text {, } \\
& \left\{\left(e_{2}, r, 0\right)=\left\{\left(\frac{u_{1}}{\langle 0,1\rangle}, 0.9\right),\left(\frac{u_{2}}{\langle 0.25,0.75\rangle}, 0.7\right),\right.\right. \\
& \left.\left.\left(\frac{u_{3}}{\langle 0.1,0.9\rangle}, 0.15\right)\right\}\right\} \text {, } \\
& \left\{\left(e_{4}, r, 0\right)=\left\{\left(\frac{u_{1}}{\langle 0.5,0.45\rangle}, 0.1\right),\left(\frac{u_{2}}{\langle 0.3,0.7\rangle}, 0.1\right),\right.\right. \\
& \left.\left.\left(\frac{u_{3}}{\langle 0.4,0.4\rangle}, 0.4\right)\right\}\right\} \text {. }
\end{aligned}
$$

Next the PIFSES $\left(F_{p}, Z\right)$ is used together with a generalized algorithm to solve the decision making problem stated at the beginning of this section. The algorithm given below is employed by the hiring committee to determine the best or most suitable candidate to be hired for the position. This algorithm is a generalization of the algorithm introduced by Alkhazaleh and Salleh (see [3]) which is used in the context of the PIFSES model that is introduced in this paper. The generalized algorithm is as follows.

Algorithm

(1) Input the PIFSES $\left(F_{p}, Z\right)$.

(2) Find the values of $\mu_{F_{p}\left(z_{i}\right)}\left(u_{i}\right)-\nu_{F_{p}\left(z_{i}\right)}\left(u_{i}\right)$ for each element $u_{i} \in U$, where $\mu_{F_{p}\left(z_{i}\right)}\left(u_{i}\right)$ and $\nu_{F_{p}\left(z_{i}\right)}\left(u_{i}\right)$ are the membership function and nonmembership function of each of the elements $u_{i} \in U$, respectively.

(3) Find the highest numerical grade for the agreePIFSES and disagree-PIFSES.

(4) Compute the score of each element $u_{i} \in U$ by taking the sum of the products of the numerical grade of each element with the corresponding degree of possibility $\mu_{i}$, for the agree-PIFSES and disagree-PIFSES, denoted by $A_{i}$ and $D_{i}$, respectively.

(5) Find the values of the score $r_{i}=A_{i}-D_{i}$ for each element $u_{i} \in U$.

(6) Determine the value of the highest score, $s=$ $\max _{u_{i} \in U}\left\{r_{i}\right\}$. Then the decision is to choose element $u_{i}$ as the optimal or best solution to the problem. If there is more than one element with the highest $r_{i}$ score, then any one of those elements can be chosen as the optimal solution.

Then we can conclude that the optimal choice for the hiring committee is to hire candidate $u_{i}$ to fill the vacant position.

Table 1 gives the values of $\mu_{F_{p}\left(z_{i}\right)}\left(u_{i}\right)-\nu_{F_{p}\left(z_{i}\right)}\left(u_{i}\right)$ for each element $u_{i} \in U$. It is to be noted that the first terms and second terms for each element in Table 1 represent the values of $\mu_{F_{p}\left(z_{i}\right)}\left(u_{i}\right)-\nu_{F_{p}\left(z_{i}\right)}\left(u_{i}\right)$ and the degree of possibility of the element $u_{i} \in U$, respectively.

Tables 2 and 3 give the highest numerical grade for the elements in the agree-PIFSES and disagree-PIFSES, respectively.

Let $A_{i}$ and $D_{i}$ represent the score of each numerical grade for the agree-PIFSES and disagree-PIFSES, respectively. These values are given in Table 4.

Then $s=\max _{u_{i} \in U}\left\{r_{i}\right\}=r_{2}$. Therefore, the hiring committee should hire candidate $u_{2}$ to fill in the vacant position.

\section{Conclusion}

In this paper the concept of possibility intuitionistic fuzzy soft expert set was established. The basic operations on possibility intuitionistic fuzzy soft expert sets, namely, the complement, union, intersection, AND, and OR operations, were defined. Subsequently, the basic properties of these operations such 
TABLE 1: Values of $\mu_{F_{p}\left(z_{i}\right)}\left(u_{i}\right)-v_{F_{p}\left(z_{i}\right)}\left(u_{i}\right)$ for all $u_{i} \in U$.

\begin{tabular}{cccc}
\hline & $u_{1}$ & $u_{2}$ & $u_{3}$ \\
\hline$\left(e_{1}, p, 1\right)$ & $-0.2,0.3$ & $0,0.2$ & $0.8,0.3$ \\
$\left(e_{2}, p, 1\right)$ & $-0.95,0.9$ & $-0.35,0.8$ & $-0.5,0.15$ \\
$\left(e_{3}, p, 1\right)$ & $-0.2,0.1$ & $0.2,0.8$ & $-0.4,0.3$ \\
$\left(e_{4}, p, 1\right)$ & $-0.7,0.9$ & $1,0.5$ & $-1,1$ \\
$\left(e_{1}, q, 1\right)$ & $0.6,0.3$ & $-0.1,0.2$ & $0.3,0.3$ \\
$\left(e_{2}, q, 1\right)$ & $0.2,0.6$ & $1,0.8$ & $0.9,0.5$ \\
$\left(e_{3}, q, 1\right)$ & $0.8,0.9$ & $0.3,0.4$ & $-0.9,0.5$ \\
$\left(e_{4}, q, 1\right)$ & $-0.8,0.7$ & $-0.45,0.25$ & $1,0.45$ \\
$\left(e_{1}, r, 1\right)$ & $-0.25,0.7$ & $-0.6,0.25$ & $-1,0$ \\
$\left(e_{2}, r, 1\right)$ & $-0.2,0.6$ & $0.35,0.95$ & $-0.2,0.1$ \\
$\left(e_{3}, r, 1\right)$ & $0.75,0.99$ & $-0.55,0.8$ & $-0.85,0.4$ \\
$\left(e_{1}, p, 0\right)$ & $-0.4,0.5$ & $-0.1,0$ & $0.9,0.1$ \\
$\left(e_{3}, p, 0\right)$ & $-0.92,0.75$ & $-0.4,0.2$ & $0.65,0.6$ \\
$\left(e_{4}, p, 0\right)$ & $0.8,0.62$ & $1,0.05$ & $-0.2,0.9$ \\
$\left(e_{1}, q, 0\right)$ & $-0.2,0.55$ & $0.4,1$ & $1,0.99$ \\
$\left(e_{2}, q, 0\right)$ & $-0.5,0.2$ & $-0.65,0.3$ & $0.1,0.1$ \\
$\left(e_{3}, q, 0\right)$ & $-1,0.6$ & $-0.35,0.8$ & $-0.9,1$ \\
$\left(e_{4}, q, 0\right)$ & 1,1 & $-0.1,0.4$ & $-0.5,0.9$ \\
$\left(e_{1}, r, 0\right)$ & $-0.2,0.4$ & $0.25,0.7$ & $-0.2,0.3$ \\
$\left(e_{2}, r, 0\right)$ & $-1,0.9$ & $-0.5,0.7$ & $-0.8,0.15$ \\
$\left(e_{4}, r, 0\right)$ & $0.05,0.1$ & $-0.4,0.1$ & $0,0.4$ \\
\hline & & &
\end{tabular}

TABLE 2: Numerical grade for agree-PIFSES.

\begin{tabular}{cccc}
\hline & $u_{i}$ & Highest numerical grade & Degree of possibility, $\mu_{i}$ \\
\hline$\left(e_{1}, p\right)$ & $u_{3}$ & 0.8 & 0.3 \\
$\left(e_{2}, p\right)$ & $u_{2}$ & -0.35 & 0.8 \\
$\left(e_{3}, p\right)$ & $u_{2}$ & 0.2 & 0.8 \\
$\left(e_{4}, p\right)$ & $u_{2}$ & 1 & 0.5 \\
$\left(e_{1}, q\right)$ & $u_{1}$ & 0.6 & 0.3 \\
$\left(e_{2}, q\right)$ & $u_{2}$ & 1 & 0.8 \\
$\left(e_{3}, q\right)$ & $u_{1}$ & 0.8 & 0.9 \\
$\left(e_{4}, q\right)$ & $u_{3}$ & 1 & 0.45 \\
$\left(e_{1}, r\right)$ & $u_{1}$ & -0.25 & 0.7 \\
$\left(e_{2}, r\right)$ & $u_{2}$ & 0.35 & 0.95 \\
$\left(e_{3}, r\right)$ & $u_{1}$ & 0.75 & 0.99 \\
\hline
\end{tabular}

Score $\left(u_{1}\right)=1.4675$; score $\left(u_{2}\right)=1.5125$; score $\left(u_{3}\right)=0.69$.

TABLE 3: Numerical grade for disagree-PIFSES.

\begin{tabular}{cccc}
\hline & $u_{i}$ & Highest numerical grade & Degree of possibility, $\mu_{i}$ \\
\hline$\left(e_{1}, p\right)$ & $u_{3}$ & 0.9 & 0.1 \\
$\left(e_{3}, p\right)$ & $u_{3}$ & 0.65 & 0.6 \\
$\left(e_{4}, p\right)$ & $u_{2}$ & 1 & 0.05 \\
$\left(e_{1}, q\right)$ & $u_{3}$ & 1 & 0.99 \\
$\left(e_{2}, q\right)$ & $u_{3}$ & 0.1 & 0.1 \\
$\left(e_{3}, q\right)$ & $u_{2}$ & -0.35 & 0.8 \\
$\left(e_{4}, q\right)$ & $u_{1}$ & 1 & 1 \\
$\left(e_{1}, r\right)$ & $u_{2}$ & 0.25 & 0.7 \\
$\left(e_{2}, r\right)$ & $u_{2}$ & -0.5 & 0.7 \\
$\left(e_{4}, r\right)$ & $u_{1}$ & 0.05 & 0.1 \\
\hline
\end{tabular}

Score $\left(u_{1}\right)=1.005$; score $\left(u_{2}\right)=-0.405$; score $\left(u_{3}\right)=1.48$.
TABle 4: The score $r_{i}=A_{i}-D_{i}$.

\begin{tabular}{lcc}
\hline$A_{i}$ & $D_{i}$ & $r_{i}$ \\
\hline Score $\left(u_{1}\right)=1.4675$ & Score $\left(u_{1}\right)=1.005$ & 0.4625 \\
Score $\left(u_{2}\right)=1.5125$ & Score $\left(u_{2}\right)=-0.405$ & 1.9175 \\
Score $\left(u_{3}\right)=0.69$ & Score $\left(u_{3}\right)=1.48$ & -0.79 \\
\hline
\end{tabular}

as De Morgan's laws and other relevant laws pertaining to the concept of PIFSES are proved. Finally, a generalized algorithm is introduced and applied to the PIFSES model to solve a hypothetical decision making problem.

\section{Conflict of Interests}

The authors declare that there is no conflict of interests regarding the publication of this paper.

\section{Acknowledgments}

The authors would like to gratefully acknowledge the financial assistance received from the Ministry of Education, Malaysia, and UCSI University, Malaysia, under Grant no. FRGS/1/2014/ST06/UCSI/03/1.

\section{References}

[1] D. Molodtsov, "Soft set theory-first results," Computers \& Mathematics with Applications, vol. 37, no. 4-5, pp. 19-31, 1999.

[2] P. K. Maji, R. Biswas, and A. R. Roy, “Fuzzy soft sets," Journal of Fuzzy Mathematics, vol. 3, no. 9, pp. 589-602, 2001.

[3] S. Alkhazaleh and A. R. Salleh, "Soft expert sets," Advances in Decision Sciences, vol. 2011, Article ID 757868, 12 pages, 2011.

[4] S. Alkhazaleh, A. R. Salleh, and N. Hassan, "Possibility fuzzy soft set," Advances in Decision Sciences, vol. 2011, Article ID 479756, 18 pages, 2011.

[5] S. Alkhazaleh, A. R. Salleh, and N. Hassan, "Fuzzy parameterized interval-valued fuzzy soft set," Applied Mathematical Sciences, vol. 5, no. 65-68, pp. 3335-3346, 2011.

[6] M. Bashir, A. R. Salleh, and S. Alkhazaleh, "Possibility intuitionistic fuzzy soft set," Advances in Decision Sciences, vol. 2012, Article ID 404325, 24 pages, 2012.

[7] N. Hassan and K. Alhazaymeh, "Vague soft expert set theory," in Proceedings of the 20th National Symposium on Mathematical Sciences: Research in Mathematical Sciences: A Catalyst for Creativity and Innovation, vol. 1522 of AIP Conference Proceedings, pp. 953-958, Putrajaya, Malaysia, April 2013.

[8] K. Alhazaymeh and N. Hassan, "Possibility vague soft set and its application in decision making," International Journal of Pure and Applied Mathematics, vol. 77, no. 4, pp. 549-563, 2012.

[9] M. Bashir and A. R. Salleh, "Fuzzy parameterized soft expert set," Abstract and Applied Analysis, vol. 2012, Article ID 258361, 15 pages, 2012.

[10] G. Selvachandran, "Possibility vague soft expert set theory," in Proceedings of the International Conference on Soft Computing in Data Science, Putrajaya, Malaysia, September 2015.

[11] K. T. Atanassov, "Intuitionistic fuzzy sets," Fuzzy Sets and Systems, vol. 20, no. 1, pp. 87-96, 1986. 
[12] P. K. Maji, R. Biswas, and A. R. Roy, "Intuitionistic fuzzy soft sets," Journal of Fuzzy Mathematics, vol. 9, no. 3, pp. 677-692, 2001.

[13] Y.-J. Xu, Y.-K. Sun, and D.-F. Li, "Intuitionistic fuzzy soft set," in Proceedings of the 2nd International Workshop on Intelligent Systems and Applications (ISA '10), pp. 1-4, May 2010.

[14] K. M. Lee, "Comparison of interval-valued fuzzy sets, intuitionistic fuzzy sets and bipolar-valued fuzzy sets," Journal of Korean Institute of Intelligent Systems, vol. 14, no. 2, pp. 125-129, 2004.

[15] K. M. Lee, "Bipolar-valued fuzzy sets and their operations," in Proceedings of the International Conference on Intelligent Technologies, pp. 307-312, Bangkok, Thailand, 2000. 


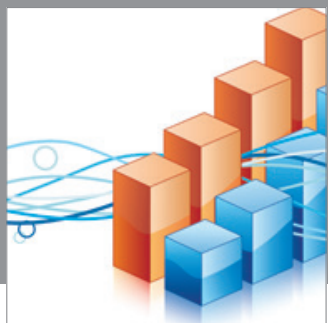

Advances in

Operations Research

mansans

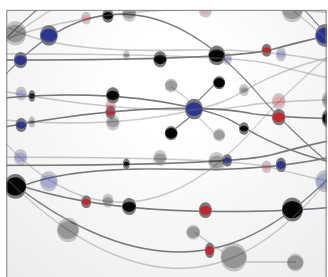

The Scientific World Journal
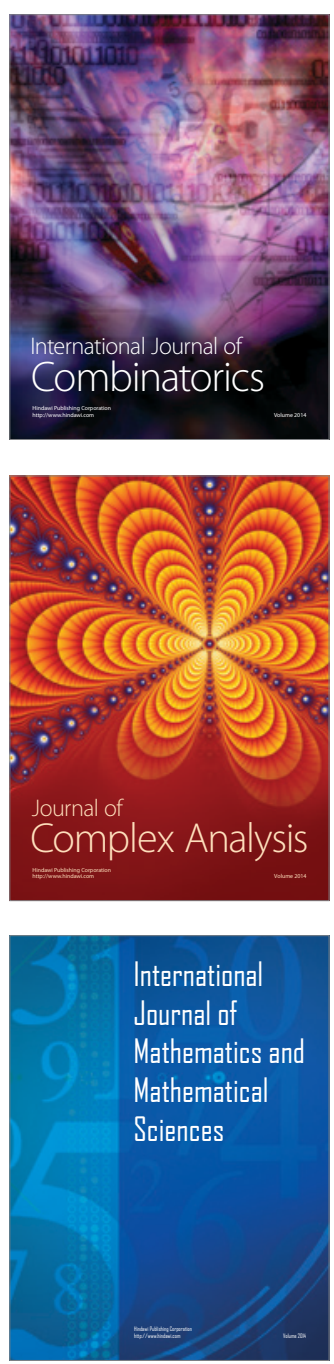
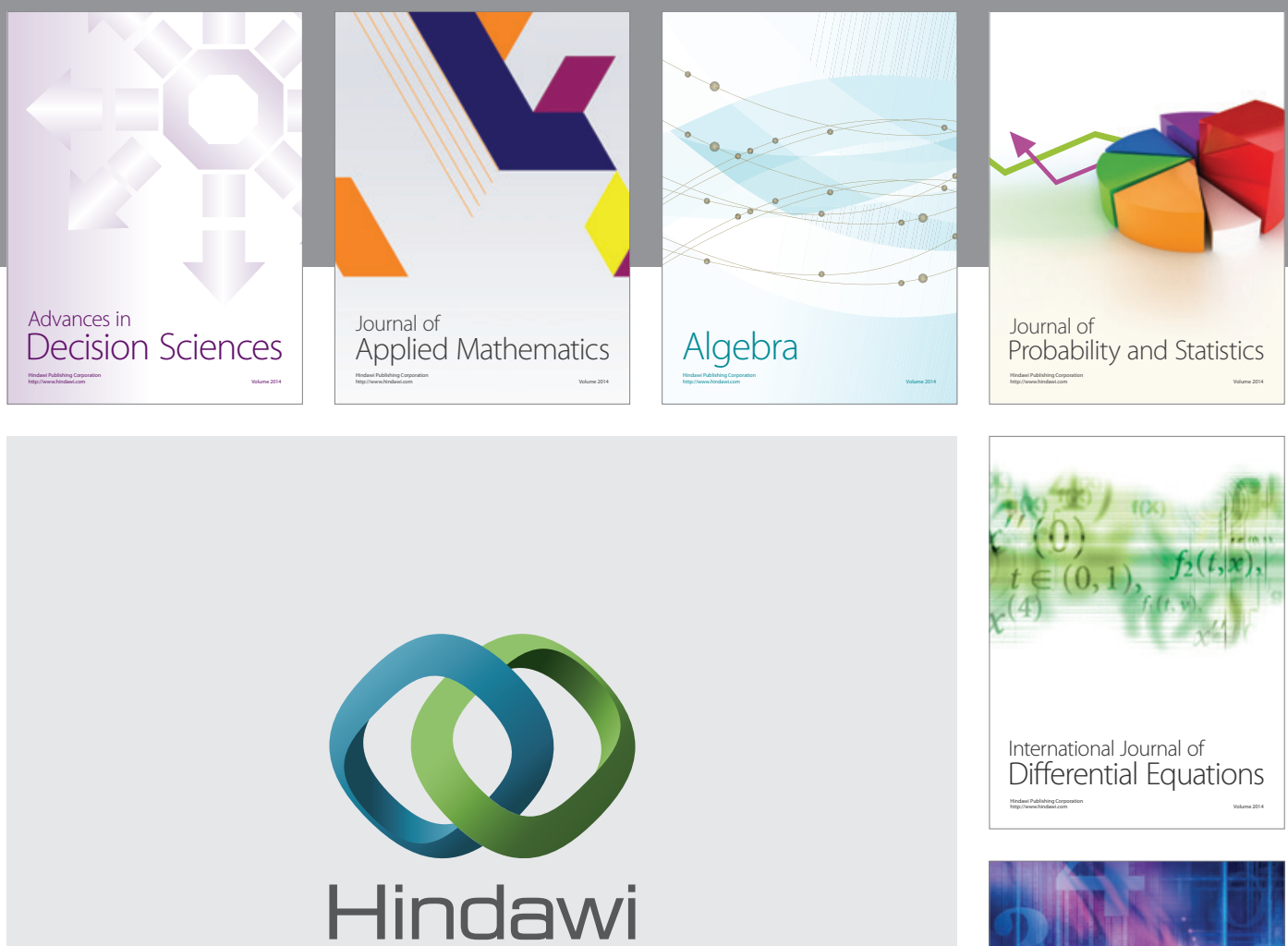

Submit your manuscripts at http://www.hindawi.com
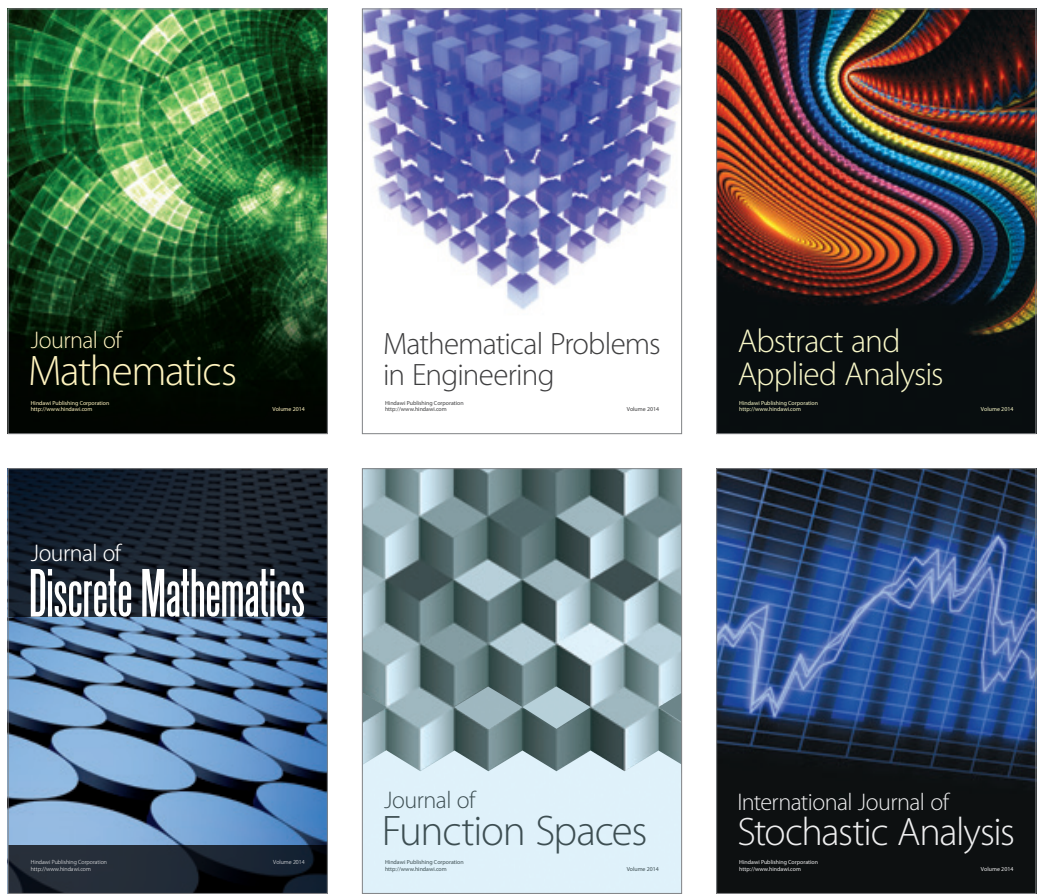

Journal of

Function Spaces

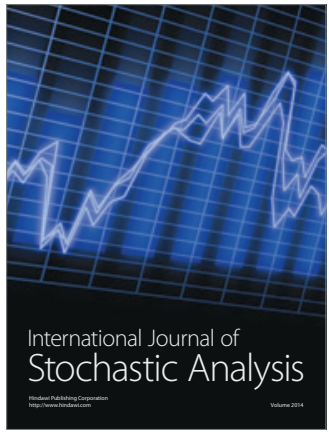

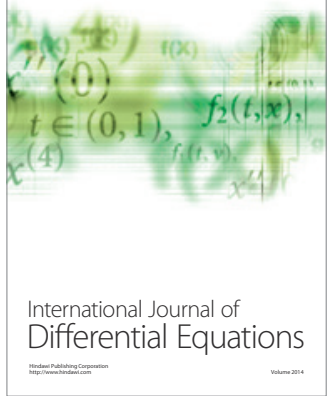
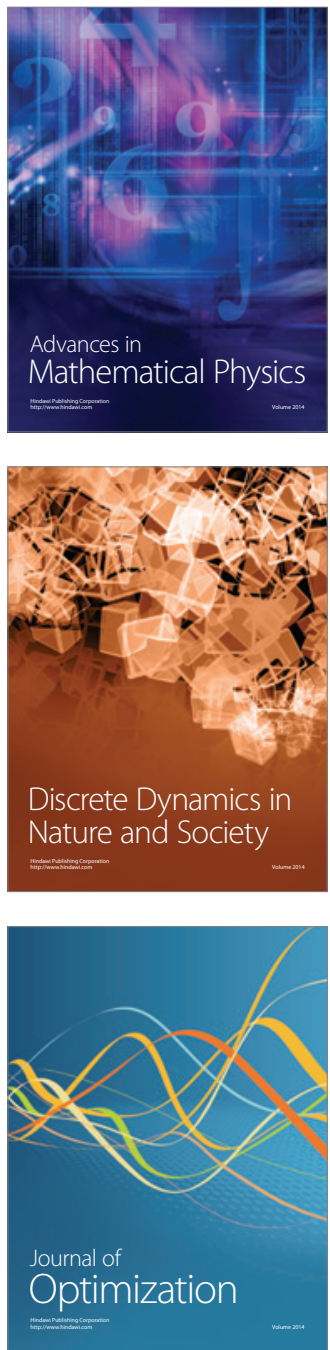